\title{
ANALYZING THE DYNAMICS OF DETERMINISTIC SYSTEMS FROM A HYPERGRAPH THEORETICAL POINT OF VIEW
}

\author{
Luis M. Torres ${ }^{1}$ And Annegret K. WAgleR ${ }^{2}$
}

\begin{abstract}
To model the dynamics of discrete deterministic systems, we extend the Petri nets framework by a priority relation between conflicting transitions, which is encoded by orienting the edges of a transition conflict graph. The aim of this paper is to gain some insight into the structure of this conflict graph and to characterize a class of suitable orientations by an analysis in the context of hypergraph theory.
\end{abstract}

Keywords. Petri nets, deterministic dynamic systems, hypergraphs.

Mathematics Subject Classification. 92C42, 68Q85, 05C65.

\section{INTRODUCTION}

For modeling complex dynamic systems, Petri nets constitute a well-established framework, see, e.g., [18] for an overview. Their broad application range includes the design of asynchronous hardware circuits [23], the analysis of production and workflow systems [2], the analysis and control of batch processes [9], the design of distributed algorithms for networks of agents [19], and the modeling and simulation of biological networks $[10,12]$, to cite some prominent examples.

Petri nets have been extended in various ways for dealing with further applications. For instance, colored and high-level Petri nets are used for protocol specification in communication networks $[5,11]$, stochastic Petri nets model the noise

Received February 1, 2012. Accepted November 15, 2013.

1 Research Center on Mathematical Modelling MODEMAT, Escuela Politécnica Nacional, Quito, Ecuador. luis.torres@epn.edu.ec

2 Laboratoire d'Informatique, de Modélisation et d'Optimisation des Systèmes (LIMOS),

Université Blaise Pascal, Clermont-Ferrand, France. Annegret.WAGLER@univ-bpclermont.fr 
coming from the input data or being intrinsic to a system [3,13], and hybrid Petri nets are used for systems were both continuous and discrete processes coexist [6]. In this paper we consider another extension introduced in [22] to model systems with a discrete event-based dynamic behavior exhibiting deterministic features. For instance, some biological systems are of this nature, as stimulating them in a certain way triggers always the same response (see e.g. the light-induced sporulation of Physarum polycephalum plasmodia or the phototaxis of halobacterial cells described in [14-16]).

In general, the structure of the studied systems is described by means of a network, while the studied dynamic processes are usually represented in terms of state changes.

The network is a weighted directed bipartite graph $G=(P \cup T, A, w)$ with two kinds of nodes, places and transitions, linked by weighted directed $\operatorname{arcs}$. The set $P$ of places represents the system's components, while the set $T$ of transitions stands for the possible interactions. We denote by $A$ the set of arcs in the network and by $w$ the vector of their weights, with $w_{i t}$ being the weight of $\operatorname{arc}(i, t) \in A$.

Some places $B \subseteq P$ have bounded capacities associated with them, which are given by a positive integral vector $u \in \mathbb{Z}_{+}^{B}$. We refer to a capacitated network by $(G, u)$.

A state ${ }^{3}$ of the system is an assignment of tokens to places that takes into account the capacities of the places in $B$. Hence, any state can be represented as an integral nonnegative vector $x \in \mathbb{Z}_{+}^{P}$ and the potential state space of the system is the set of all theoretically possible states:

$$
\mathcal{X}:=\left\{x \in \mathbb{Z}_{+}^{P}: x_{i} \leq u_{i}, \forall i \in B\right\} .
$$

Observe that $\mathcal{X}$ is finite if $B=P$ holds.

Dynamic processes are described as sequences $x^{1}, \ldots, x^{k}$ of consecutive system states $^{4}$, where state $x^{i+1}$ is obtained from $x^{i}$ by switching a transition $t \in T$. Thereby, $t$ consumes $w_{i t}$ tokens from each pre-place $i$ in

$$
P^{-}(t):=\{i \in P:(i, t) \in A\}
$$

and produces $w_{t i}$ new tokens on each post-place $i$ in

$$
P^{+}(t):=\{i \in P:(t, i) \in A\} .
$$

A transition $t \in T$ is enabled at a state $x \in \mathcal{X}$ if switching $t$ yields a valid successor state and disabled otherwise. Thus, the set of enabled transitions at state $x$ is

$$
T(x):=\left\{t \in T: x_{i}-w_{i t} \geq 0, \forall i \in P^{-}(t) ; x_{i}+w_{t i} \leq u_{i}, \forall i \in P^{+}(t) \cap B\right\} .
$$

A Petri net is a pair $\left(G, x^{0}\right)$ consisting of a network $G$ together with an initial state $x^{0}$, and its state space is understood as the set of all further system states

\footnotetext{
${ }^{3}$ A state is also called marking $m: P \rightarrow \mathbb{Z}_{+}$in the Petri net community.

${ }^{4}$ Throughout this paper, we will use superindices to reference different states and subindices to specify places. Thus, $x_{i}^{j}$ is the number of tokens assigned to place $i$ at state $x^{j}$.
} 
which can be reached from $x^{0}$ by switching sequences of transitions. Describing the dynamics might be done by model animation, i.e., by simulating the flow of tokens inside the network.

If $T(x)$ contains more than one transition for some state $x$, a decision between the alternatives is taken non-deterministically. This leads to a branching system behavior, and describing dynamic processes is done in terms of potential reachability with the help of model animation and simulation.

In particular, it is not possible to model deterministic systems, where any state $x \in \mathcal{X}$ has a unique successor state $\operatorname{succ}(x)$. Hence, if there is a state $x$ in such a system where more than one transition is enabled, a deterministic decision is taken in order to select a unique transition trans $(x) \in T(x)$ that must be switched in order to reach $\operatorname{succ}(x)$.

An explicit encoding of trans $(x)$, for instance state-wise, is at least exponential in the size of $G$ and $u$. In [22] we proposed a compact encoding, using orientations of the following conflict graph. The transition conflict graph of $G$ is an undirected graph $\mathbb{K}=(T, \mathbb{E})$ having as nodes the transitions from $G$, where two transitions $t, t^{\prime}$ are joined by an edge if and only if there exists at least one state where both are enabled, i.e., $t t^{\prime} \in \mathbb{E} \Leftrightarrow X(t) \cap X\left(t^{\prime}\right) \neq \emptyset$, where

$$
X(t):=\left\{x \in \mathcal{X}: x_{i} \geq w_{i t}, \forall i \in P^{-}(t) ; x_{i} \leq u_{i}-w_{t i}, \forall i \in P^{+}(t) \cap B\right\}
$$

denotes the set of states at which transition $t$ is enabled. $\mathbb{K}$ can be constructed in $O\left(|P||T|^{2}\right)$ time using a straightforward algorithm to check for box intersections or in a more efficient way suggested in [22]. It clearly follows that, for every state $x \in \mathcal{X}$, the set $T(x)$ induces a clique in $\mathbb{K}$, i.e., a set of mutually adjacent nodes.

A directed graph $\mathbb{D}$ obtained by orienting the edges of $\mathbb{K}$ is said to be a valid orientation if, for every state $x \in \mathcal{X}$ with $T(x) \neq \emptyset$, the clique induced by the nodes from $T(x)$ has a unique sink, and this sink coincides with $\operatorname{trans}(x)$. The arcs of $\mathbb{D}$ can be interpreted as priority relations between pairs of transitions, with $\operatorname{trans}(x)$ being the transition with highest priority in $T(x)$ [17]. This allows us to explore the dynamic system behavior including reachability questions with the help of successor or predecessor oracles based on determining $\operatorname{trans}(x)$ [22]. Note that the oracles are encoded in a compact way as the size of $\mathbb{D}$ is $O\left(|T|^{2}\right)$.

In [22], it is shown that for any undirected graph $H$, there is a network $G$ having $H$ as its transition conflict graph. Thus, in general, neither $\mathbb{K}$ nor $\mathbb{D}$ show any particular structural properties from a graph-theoretical point of view, which turns characterizing valid orientations into a difficult task. The aim of this paper is to provide some insights into the structural properties of special classes of transition conflict graphs and their valid orientations in the context of hypergraph theory.

\section{Hypergraphs RElated TO THE TRANSition CONFLiCT GRAPH}

Let $G=(P \cup T, A, w)$ be a network and $\mathbb{K}=(T, \mathbb{E})$ its transition conflict graph. By definition, for every state $x \in \mathcal{X}$, the set $T(x)$ of enabled transitions induces a clique in $\mathbb{K}$. The converse is not necessarily true (see [22] for an example), but 
we show that at least the inclusion-wise maximal cliques in $\mathbb{K}$ are associated with states of the system.

For that, consider the following equivalence relation on $\mathcal{X}$ :

$$
x \sim x^{\prime} \quad \Leftrightarrow \quad T(x)=T\left(x^{\prime}\right) .
$$

This relation partitions the state space into $r \leq 2^{|T|}$ equivalence classes $\mathcal{X}_{1}, \ldots, \mathcal{X}_{r}$ of states that share the same sets of enabled transitions. Let $\tilde{x}^{i} \in \mathcal{X}_{i}$ with $1 \leq i \leq r$ be (arbitrarily chosen) representative elements for each of these classes, and define the transition hypergraph $\mathcal{H}_{T}:=\left(T, \mathcal{E}_{T}\right)$ of $G$ to be the hypergraph on the set $T$ of transitions, whose family $\mathcal{E}_{T}$ of hyperedges is given by

$$
\mathcal{E}_{T}:=\left\{T\left(\tilde{x}^{i}\right): 1 \leq i \leq r\right\} .
$$

Thus, the size of $\mathcal{H}_{T}$ is exponential in the number of transitions of the network, but it is always finite, as there are at most $2^{|T|}$ different subsets of $T$.

The dual hypergraph $\mathcal{H}_{\tilde{\mathcal{X}}}$ of $\mathcal{H}_{T}$ has $\tilde{\mathcal{X}}:=\left\{\tilde{x}^{1}, \ldots, \tilde{x}^{r}\right\}$ as node set, and its family of hyperedges is determined by

$$
\mathcal{E}_{\tilde{\mathcal{X}}}:=\{X(t) \cap \tilde{\mathcal{X}}: t \in T\} .
$$

We call $\mathcal{H}_{\tilde{\mathcal{X}}}$ the state hypergraph of $G$.

A hypergraph $\mathcal{H}:=(V, \mathcal{E})$ has the Helly property if, for any family $\mathcal{E}^{\prime} \subseteq \mathcal{E}$ of pairwise intersecting hyperedges, there exists at least one node $v \in V$ contained in all hyperedges from $\mathcal{E}^{\prime}$. A well-known result in hypergraph theory [4] states that $\mathcal{H}$ has the Helly property if and only if the inclusionwise maximal hyperedges of its dual hypergraph $\mathcal{H}^{*}$ are precisely the inclusionwise maximal cliques of the intersection graph $G(\mathcal{H})$ of $\mathcal{H}$. The nodes of $G(\mathcal{H})$ represent the hyperedges of $\mathcal{H}$, and two nodes are joined by an edge if and only if the corresponding hyperedges intersect. exactly $G\left(\mathcal{H}_{\tilde{\mathcal{X}}}\right)$. Observe that in our case we have:

Observation 2.1. For a capacitated network $(G, u)$, the transition conflict graph $\mathbb{K}$ is exactly the intersection graph $G\left(\mathcal{H}_{\tilde{\mathcal{X}}}\right)$ of the state hypergraph $\mathcal{H}_{\tilde{\mathcal{X}}}$.

Moreover, we have the following result.

Lemma 2.2. For a capacitated network $(G, u)$, the state hypergraph $\mathcal{H}_{\tilde{\mathcal{X}}}$ satisfies the Helly property.

Proof. Consider the hypergraph $\mathcal{H}_{\mathcal{X}}:=\left(\mathcal{X}, \mathcal{E}_{\mathcal{X}}\right)$, which has one node for each potential state and a set of hyperedges defined by

$$
\mathcal{E}_{\mathcal{X}}:=\{X(t): t \in T\} .
$$

Observe that $\mathcal{H}_{\mathcal{X}}$ may be infinite (if some places of the network are unbounded) and that each hyperedge is a box in the lattice $\mathcal{X}$. Families of boxes in integral lattices are one classical example of hypergraphs that satisfy the Helly property. We show that this property is transferred to $\mathcal{H}_{\tilde{\mathcal{X}}}$. 
Let $\mathcal{E}_{\tilde{\mathcal{X}}}^{\prime}:=\left\{X\left(t_{1}\right) \cap \tilde{\mathcal{X}}, \ldots, X\left(t_{k}\right) \cap \tilde{\mathcal{X}}\right\}$ be a family of pairwise intersecting hyperedges in $\mathcal{H}_{\tilde{\mathcal{X}}}$. Consequently, $\mathcal{E}_{\mathcal{X}}^{\prime}:=\left\{X\left(t_{1}\right), \ldots, X\left(t_{k}\right)\right\}$ is a family of pairwise intersecting hyperedges in $\mathcal{H}_{\mathcal{X}}$ and due to the Helly property there exists a state $x \in \mathcal{X}$ with $x \in X\left(t_{i}\right), \forall 1 \leq i \leq k$. Now recall the partition of $\mathcal{X}$ introduced by the equivalence relation $(2.1)$ and let $\mathcal{X}_{\ell}$ be the equivalence class containing $x$. Then $T(x)=T\left(\tilde{x}^{\ell}\right)$ holds for the corresponding representative element $\tilde{x}^{\ell}$, and hence for any $t \in T$ we have $x \in X(t)$ if and only if $\tilde{x}^{\ell} \in X(t)$. Since $\tilde{x}^{\ell} \in \tilde{\mathcal{X}}$ holds by definition,

$$
\tilde{x}^{\ell} \in X\left(t_{i}\right) \cap \tilde{\mathcal{X}}, \forall 1 \leq i \leq k
$$

finally follows.

Combining Lemma 2.2 and the preceding observations, we obtain:

Theorem 2.3. Consider a capacitated network with transition conflict graph $\mathbb{K}$ and transition hypergraph $\mathcal{H}_{T}=\left(T, \mathcal{E}_{T}\right)$. The inclusionwise maximal hyperedges from $\mathcal{E}_{T}$ are exactly the inclusionwise maximal cliques of $\mathbb{K}$. Thus, for every inclusionwise maximal clique $Q$ of $\mathbb{K}$ there is some state $\tilde{x}^{i} \in \tilde{\mathcal{X}}, 1 \leq i \leq r$, satisfying $T\left(\tilde{x}^{i}\right)=Q$.

A natural question is whether the studied transition conflict graphs have a special structure from a graph-theoretical point of view. As it is shown in [22], this is not the case, since for any arbitrary undirected graph $H$, there exists a network $(G, u)$ with the property that its transition conflict graph $\mathbb{K}$ is isomorphic to $H$. We point out in the following that, however, $\mathbb{K}$ can have some interesting properties, provided that $\mathcal{H}_{T}$ and $\mathcal{H}_{\tilde{\mathcal{X}}}$ belong to certain classes of hypergraphs.

A cycle of length $k$ in a hypergraph $\mathcal{H}=(V, \mathcal{E})$ is a sequence

$$
\left(v_{1}, E_{1}, v_{2}, E_{2}, \ldots, v_{k}, E_{k}, v_{k+1}\right)
$$

such that $v_{i} \in V$ for all $1 \leq i \leq k+1, E_{i} \in \mathcal{E}$ for all $1 \leq i \leq k, E_{i} \neq E_{j}$ if $i \neq j$, $v_{i}, v_{i+1} \in E_{i}$ for all $1 \leq i \leq k$, and $v_{k+1}=v_{1}$.

A hyperedge $E_{1} \in \mathcal{H}=(V, \mathcal{E})$ is called an ear if there exists another hyperedge $E_{2} \in \mathcal{H}$ such that, for any $v \in E_{1} \backslash E_{2}$, no hyperedge different from $E_{1}$ contains $v$. The operation of removing the ear $E$ consists in deleting $E$ from $\mathcal{E}$ and deleting from $V$ all nodes that are only contained in $E$. A hypergraph is acyclic if it can be reduced to the empty hypergraph by a sequence of ear removals.

In contrast to the analogue concepts in graph theory, a hypergraph can contain a cycle and be acyclic at the same time. For instance, let $V:=\{1, \ldots, 6\}$ and $\mathcal{E}:=\left\{E_{1}, E_{2}, E_{3}\right\}$, with $E_{i}:=\{1,2,3,3+i\}$, for $1 \leq i \leq 3$. One can check that the hypergraph $\mathcal{H}=(V, \mathcal{E})$ contains the cycle $1, E_{1}, 2, E_{2}, 3, E_{3}, 1$. At the same time, $E_{1}, E_{2}, E_{3}$ provides a valid sequence of ear removals that reduces $\mathcal{H}$ to the empty hypergraph, so $\mathcal{H}$ is acyclic. 
According to [1], a hypergraph $\mathcal{H}$ is acyclic if and only if its dual $\mathcal{H}^{*}$ has the Helly property, and for every cycle of length at least 3 in $\mathcal{H}$, some edge of $\mathcal{H}$ contains at least 3 nodes of the cycle. This implies together with the previous results:

Lemma 2.4. For a capacitated network $(G, u)$, the transition hypergraph $\mathcal{H}_{T}$ is acyclic if and only if for every cycle of length at least 3 in $\mathcal{H}_{T}$, there exists a state $\tilde{x} \in \tilde{\mathcal{X}}$ such that $T(\tilde{x})$ contains at least three nodes (i.e., transitions of $G$ ) of the cycle.

A hypergraph $\mathcal{H}^{*}=\left(V^{*}, \mathcal{E}^{*}\right)$ is called arboreal if there exists a tree $T^{*}$ on the node set $V^{*}$ such that every hyperedge of $\mathcal{H}^{*}$ induces a subtree in $T^{*}$. Due to structural characterizations in $[7,8,20]$, arboreal hypergraphs are dual to acyclic hypergraphs.

Moreover, $\mathcal{H}^{*}$ is arboreal if and only if $\mathcal{H}^{*}$ has the Helly property and every cycle of length at least 3 contains three intersecting edges. In our case we obtain:

Lemma 2.5. For a capacitated network $(G, u)$, the state hypergraph $\mathcal{H}_{\tilde{\mathcal{X}}}$ is arboreal if and only if for every cycle of length at least 3 in $\mathcal{H}_{\tilde{\mathcal{X}}}$, there exist a state $\tilde{x} \in \tilde{\mathcal{X}}$ and (at least) three hyperedges $X\left(t_{1}\right), X\left(t_{2}\right), X\left(t_{3}\right)$ in the cycle such that $\tilde{x} \in X\left(t_{1}\right) \cap X\left(t_{2}\right) \cap X\left(t_{3}\right)$.

Observe that the condition $\tilde{x} \in X\left(t_{1}\right) \cap X\left(t_{2}\right) \cap X\left(t_{3}\right)$ is equivalent to $\left\{t_{1}, t_{2}, t_{3}\right\} \subset T(\tilde{x})$.

A graph is called chordal if each of its cycles having four or more nodes contains a chord, i.e., an edge joining two nodes that are not adjacent in the cycle. Arboreal hypergraphs and chordal intersection graphs are closely related, as shown in the next result.

Lemma 2.6. A hypergraph $\mathcal{H}^{*}$ is arboreal if and only if $\mathcal{H}^{*}$ has the Helly property and its intersection graph $G\left(\mathcal{H}^{*}\right)$ is chordal.

Proof. Assume $\mathcal{H}^{*}=(V, \mathcal{E})$ is arboreal and let $E_{1}, \ldots, E_{k}$, with $k \geq 4$, be (the nodes of) a cycle $C$ in the intersection graph $G\left(\mathcal{H}^{*}\right)$. Then $E_{i} \cap E_{i+1} \neq \emptyset$ holds for $1 \leq i<k$, and $E_{1} \cap E_{k} \neq \emptyset$. Thus, there exist $v_{1}, \ldots, v_{k} \in V$ with $v_{i} \in$ $E_{i-1} \cap E_{i}, \forall 1<i \leq k$ and $v_{1} \in E_{1} \cap E_{k}$. The sequence $v_{1}, E_{1}, \ldots, v_{k}, E_{k}, v_{1}$ is a cycle in $\mathcal{H}$. From the characterization above, this cycle must contain three intersecting hyperedges, which induce a clique in $G\left(\mathcal{H}^{*}\right)$. At least one edge of this clique must be a chord in $C$. Moreover, we know (again from the characterization above) that $\mathcal{H}^{*}$ has the Helly property.

Conversely, assume $\mathcal{H}^{*}$ has the Helly property and $G\left(\mathcal{H}^{*}\right)$ is chordal. Let $v_{1}, E_{1}, \ldots, v_{k}, E_{k}, v_{1}$ be a cycle in $\mathcal{H}$, with $k \geq 3$. Then $E_{1}, \ldots, E_{k}$ are the nodes of a cycle in $G\left(\mathcal{H}^{*}\right)$. Since $G\left(\mathcal{H}^{*}\right)$ is chordal, it is easy to show that this latter cycle contains a clique of size 3 . But then, the hyperedges corresponding to the nodes in this clique must be pairwise intersecting, and due to the Helly property they must share a common node. From the characterization above, $\mathcal{H}^{*}$ is arboreal. 
Since $\mathcal{H}_{\tilde{\mathcal{X}}}$ has the Helly property by Lemma 2.2, this hypergraph is arboreal if and only if $G\left(\mathcal{H}_{\tilde{\mathcal{X}}}\right)=\mathbb{K}$ is chordal. We summarize the last observations as follows.

Theorem 2.7. For a capacitated network $(G, u)$, the following assertions are equivalent:

- $\mathcal{H}_{T}$ is acyclic.

- $\mathcal{H}_{\tilde{\mathcal{X}}}$ is arboreal.

- For each sequence $t_{1}, T\left(\tilde{x}_{1}\right), t_{2}, T\left(\tilde{x}_{2}\right), \ldots, t_{k}, T\left(\tilde{x}_{k}\right), t_{1}$, with $k \geq 3, t_{i}, t_{i+1} \in$ $T\left(\tilde{x}_{i}\right) \forall 1 \leq i<k$, and $t_{1}, t_{k} \in T\left(\tilde{x}_{k}\right)$, there exists some state $\tilde{x}$ such that at least three transitions from $\left\{t_{1}, \ldots, t_{k}\right\}$ are enabled at $\tilde{x}$.

- For each sequence $\tilde{x}_{1}, X\left(t_{1}\right), \tilde{x}_{2}, X\left(t_{2}\right), \ldots, \tilde{x}_{k}, X\left(t_{k}\right), \tilde{x}_{1}$, with $k \geq 3, \tilde{x}_{i}, \tilde{x}_{i+1} \in$ $X\left(t_{i}\right) \forall 1 \leq i<k$, and $\tilde{x}_{1}, \tilde{x}_{k} \in X\left(t_{k}\right)$, there exists some state $\tilde{x}$ such that at least three transitions from $\left\{t_{1}, \ldots, t_{k}\right\}$ are enabled at $\tilde{x}$.

- $\mathbb{K}$ is chordal.

\section{VALID ORIENTATIONS}

Our purpose is to encode the dynamics of a given deterministic system $(G, u)$ in a compact way, by introducing enough priority relations among transitions, as to be able to determine $\operatorname{trans}(x)$ for every $x \in \mathcal{X}$. As the set of enabled transitions at each state corresponds to a clique in the associated transition conflict graph $\mathbb{K}$, this graph is a plausible candidate for a framework where these priorities could be embedded. This idea motivated the following definition from [22].

Let $\mathbb{D}=(T, \mathbb{A})$ be a directed graph obtained by orienting the edges of $\mathbb{K}$. Then $\mathbb{D}$ is said to be valid if, for every state $x \in \mathcal{X}$ with $T(x) \neq \emptyset$, the subgraph induced by the nodes from $T(x)$ has a unique sink, and this sink coincides with $\operatorname{trans}(x)$.

Observe that the existence of a valid orientation implicitly imposes a further requirement on the nature of a dynamic system. Namely, if two states $x, x^{*} \in \mathcal{X}$ have the same set $T(x)=T\left(x^{*}\right)$ of enabled transitions, then both induce the same subgraph of $\mathbb{D}$ and, therefore, $\operatorname{trans}(x)=\operatorname{trans}\left(x^{*}\right)$ must hold. Moreover, if a transition $t$ is enabled at two states $x, x^{\prime} \in \mathcal{X}$ and $t$ is the highest-priority transition at $x$, then either $t$ is also the highest-priority transition at $x^{\prime}$ or $\operatorname{trans}\left(x^{\prime}\right) \notin T(x)$. In $[22]$ it is shown that this condition is also sufficient for the existence of a valid orientation.

The fact that one has to check, for each clique $Q$ in $\mathbb{K}$, if there is a corresponding state $x \in \mathcal{X}$ with $T(x)=Q$ makes the recognition of valid orientations hard in general, as there might be up to $2^{|T|}$ cliques. In the following we describe a special class of orientations for which this recognition problem can be solved efficiently.

Observe that two different dynamic systems might share the same transition conflict graph and let us define the following equivalence relation between capacitated networks:

$$
(G, u) \sim_{\mathbb{K}}\left(G^{\prime}, u^{\prime}\right) \quad \Leftrightarrow \quad \mathbb{K}_{(G, u)} \cong \mathbb{K}_{\left(G^{\prime}, u^{\prime}\right)},
$$


where $\cong$ stands for graph isomorphism. An orientation is said to be strongly valid if it is valid for all networks of some equivalence class of $\sim_{\mathbb{K}}$. According to [22], strongly valid orientations are characterized as follows:

Theorem $3.1([22])$. An orientation $\mathbb{D}$ of the transition conflict graph of a capacitated network $(G, u)$ is strongly valid if and only if it does not contain any directed cycle of length 3.

Hence, strongly valid orientations can be easily recognized with the help of this characterization. Note that acyclic orientations are one specific example of strongly valid orientations, but there exist deterministic systems for which the valid orientation encoding the dynamic behavior contains directed cycles [22].

In the previous section, we considered networks for which the transition hypergraph $\mathcal{H}_{T}$ is acyclic. Theorem 2.7 states that in such cases the transition conflict graph is chordal. Moreover, note that a chord $\left(t_{i}, t_{j}\right)$ within a directed cycle $C$ induces a new directed cycle with a smaller number of nodes, as $C$ contains both a directed path from $t_{i}$ to $t_{j}$ and a directed path from $t_{j}$ to $t_{i}$. Hence, an orientation of a chordal graph contains a directed cycle of length 3 if and only if it contains any directed cycle. This observation can be used to obtain a new characterization of acyclic transition hypergraphs of capacitated networks:

Theorem 3.2. The transition hypergraph $\mathcal{H}_{T}$ of a capacitated network $(G, u)$ is acyclic if and only if, for any orientation $\mathbb{D}$ of $\mathbb{K}$, the following two statements are equivalent:

(i) $\mathbb{D}$ is strongly valid.

(ii) $\mathbb{D}$ is acyclic.

Proof. If $\mathcal{H}_{T}$ is an acyclic hypergraph, then $\mathbb{K}$ is chordal and it follows from the remarks above that $(i)$ and $(i i)$ are equivalent. Conversely, assume that for some capacitated network $(G, u)$, we have equivalence between these two statements. From Theorem 3.1, this means that $\mathbb{D}$ contains a directed cycle only if it contains a directed cycle of length 3 . Hence, $\mathbb{K}$ must be chordal and Theorem 2.7 asserts that $\mathcal{H}_{T}$ is acyclic.

\section{Conclusions}

We have examined a new approach for encoding the dynamic behavior of certain deterministic discrete systems that relies on extending the familiar framework of Petri nets. Our encoding consists in a realization of the successor-oracle as a valid orientation of the edges of the transition conflict graph $\mathbb{K}$. This encoding is compact in the sense that the amount of space required for its storage is polynomial in the size of the network. Therefore, it is well-suited for being integrated into simulation algorithms to study the dynamics of large complex deterministic systems, and to address issues such as reachability, boundedness, existence of deadlocks, and liveness, among others. 
In some applications, for instance in systems biology, the set of rules governing the dynamic behavior of the system (i.e., the valid orientation in our model) is not known a priory, but has to be reconstructed from information provided by experimentally observing some system states together with their successors. One key task for the design of the required experiments consists in finding minimum cardinality test sets, i.e., sets of system states whose experimental observation provides enough information on a partial orientation of the edges in $\mathbb{K}$ in order to infer the orientation of the remaining edges [21]. The studied test sets of states correspond to minimum cardinality transversals in the state hypergraph $\mathcal{H}_{\tilde{\mathcal{X}}}$ of the system and are hard to find in general.

For the biological systems studied in [17], the reconstructed priority relations reflect reaction rates and turned out to be partial orders on $T$ which correspond to acyclic orientations of the transition conflict graph $\mathbb{K}$. In the light of Theorem 3.2, we expect that many biological systems admit the combinatorial properties studied here. Thus the experimental design can benefit from the structure of arboreal state hypergraphs or chordal transition conflict graphs, see Theorem 2.7. In fact, each non-complete chordal graph has 2 non-adjacent simplicial nodes (i.e. nodes having a clique as neighborhood). Consecutively removing simplicial nodes from a chordal graph results in a well-known elimination scheme. The cliques forming the neighborhoods of the studied simplicial nodes are maximal and, hence, correspond to sets $T(x)$ of some states $x$ by Theorem 2.3. Subject of further research is whether and to which extent the knowledge on the successors for these states can be exploited to completely infer a valid orientation of the transition conflict graph $\mathbb{K}$. In the affirmative case, the elimination scheme for $\mathbb{K}$ would help to determine a sufficient test set of states whose experimental observation provides the required information on the set of rules governing the dynamic behavior of the studied system.

\section{REFERENCES}

[1] B.D. Acharya and M.Las Vergnas, Hypergraphs with cyclomatic number zero, triangulated hypergraphs and an inequality. J. Combin. Theory (B) 33 (1982) 52-56.

[2] N.R. Adam, V. Atluri and W.K. Huang, Modeling and analysis of workflows using Petri nets. J. Intell. Inf. Syst. 10 (1998) 131-158.

[3] G. Balbo, Introducation to stochastic Petri nets, in Lectures on formal methods and performance analysis: first EEF/Euro summer school on trends in computer science, SpringerVerlag New York, Inc., New York, NY, USA (2002) 84-155.

[4] C. Berge and P. Duchet, A generalisation of Gilmore's theorem, in Recent advances in graph theory, edited by M. Fiedler, Acad. Praha, Prague (1975) 49-55.

[5] J. Billington, M. Diaz and G. Rozenberg, Application of Petri nets to communication networks, Advances in Petri Nets. Springer-Verlag, London, UK (1999)

[6] R. David and H. Alla, Discrete, Continuous, and hybrid Petri nets. Springer-Verlag Berlin Heidelberg, Heidelberg (2005).

[7] P. Duchet, Propriété de helly et problèmes de représentations, in Problèmes Combinatoires et Théorie des Graphes, Coll. Orsay 1976, CNRS Paris (1978) 117-118.

[8] C. Flament, Hypergraphes arborés. Discrete Math. 21 (1978) 223-226. 
[9] T. Gu and P.A. Bahri, A survey of Petri net applications in batch processes. Comput. Ind. 47 (2002) 99-111.

[10] S. Hardy and P.N. Robillard, Modeling and simulation of molecular biology systems using Petri nets: modeling goals of various approaches. J. Bioinform. Comput. Biol. 2 (2004) 619-637.

[11] K.Jensen, Coloured Petri nets: basic concepts, analysis methods and practical use, vol. 3, Springer-Verlag New York, Inc., New York, NY, USA (1997)

[12] I. Koch and M. Heiner, Petri nets, in Analysis of biological networks, edited by B.H. Junker and F. Schreiber, Wiley Book Series in Bioinformatics (2008) 139-180.

[13] M. Marsan, G. Balbo, S. Donatelli, G. Franceschinis and G. Conte, Modelling with generalized stochastic Petri nets. Wiley Series in Parallel Computing (1995).

[14] W. Marwan, Theory of time-resolved somatic complementation and its use for the analysis of the sporulation control network of Physarum polycephalum. Genetics 164 (2003) 105-115.

[15] W. Marwan and C. Starostzik, The sequence of regulatory events in the sporulation control network of Physarum polycephalum analysed by time-resolved somatic complementation of mutants. Protist 153 (2002) 391-400.

[16] W. Marwan, A. Sujatha and C. Starostzik, Reconstructing the regulatory network controlling commitment and sporulation in Physarum polycephalum based on hierarchical Petri net modeling and simulation. J. Theor. Biol. 236 (2005) 349-365.

[17] W. Marwan, A. Wagler and R. Weismantel, A mathematical approach to solve the network reconstruction problem. Math. Meth. Oper. Res. 67 (2008) 117-132.

[18] W. Reisig, Petri nets: an introduction. Springer-Verlag New York, Inc., New York, NY, USA (1985).

[19] W. Reisig, Elements of distributed algorithms: modeling and analysis with Petri nets. Springer-Verlag New York, Inc., New York, NY, USA (1998).

[20] P.J. Slater, A characterization of soft hypergraphs. Canad. Math. Bull. 21 (1978) 335-337.

[21] L.M. Torres and A. Wagler, Model reconstruction for discrete deterministic systems. Electronic Notes of Discrete Mathematics 36 (2010) 175-182.

[22] L.M. Torres and A. Wagler, Encoding the dynamics of deterministic systems. Math. Methods Operations Res. 73 (2011) 281-300.

[23] A. Yakovlev, A. Koelmans, A. Semenov and D. Kinniment, Modelling, analysis and synthesis of asynchronous control circuits using Petri nets. Integr. VLSI J. 21 (1996) 143-170. 